\title{
Mechanical Overload Regulates Osteoblast Proliferation, Differentiation And Mineralization Through Wnt/B-Catenin Signaling Pathway
}

\section{Yaru Fan}

Tianjin Medical University

\section{Yingbin Yan}

The Affiliated Stomatological Hospital of Nankai University

Han Liu

The Affiliated Stomatological Hospital of Nankai University

Rui Luo

The Affiliated Stomatological Hospital of Nankai University

Hao Liu

The Affiliated Stomatological Hospital of Nankai University

\section{Yingjie Liu}

Zhang jia jie People's Hospital

\section{Ruixin Li ( $\square$ limxinxin@163.com )}

The Affiliated Stomatological Hospital of Nankai University

\section{Research Article}

Keywords: Overload, Osteoblast, Wnt signaling, Cytoskeleton

Posted Date: December 21st, 2021

DOI: https://doi.org/10.21203/rs.3.rs-1136547/v1

License: (9) This work is licensed under a Creative Commons Attribution 4.0 International License. Read Full License 


\section{Abstract}

Objectives: To study the effect of mechanical overload stimulation on proliferation, differentiation and mineralization of osteoblast and the underlying mechanisms.

Methods: MC3T3-E1 cells were divided into overload group and control group. Four-point bending loading device was used to exert mechanical overload stimulation on MC3T3-E1 cells for a certain time. The proliferation of osteoblasts was detected by MTT colorimetric assay. Real-time PCR and Western Blot were used to detect the transcription and expression of osteoblast marker genes and proteins. The specific fluorescent dyes were used to label the actin filament and the nucleus, and the changes of cytoskeleton were observed under laser scanning confocal microscope. The mineralization of osteoblasts was evaluated by the number of calcium nodules formed by alizarin red staining.

Results: Compared with the control group, the mechanical overload group significantly inhibited the proliferation of osteoblasts $(p<0.05)$. Real-time PCR and Western Blot showed that the expression of osteoblast differentiation marker gene and protein was inhibited by mechanical overload stimulation. Under laser confocal microscopy, the overload group cell shrinkage deformation was observed, also the microfilament arrangement disorder, the skeleton arrangement loose, the direction difference and the skeleton breakage, but the nucleus does not have obvious change. Alizarin red staining showed that mechanical overload inhibited the formation of calcium nodules in osteoblasts. The expression of $\beta$ catenin protein in Wnt signaling pathway was inhibited by overload mechanical stimulation under immunofluorescence microscopy.

Conclusion: Mechanical overload stimulation reduces the expression of Runx 2 by affecting the classical $W n t / \beta$-catenin signaling pathway, thus it was inhibited osteoblast proliferation, differentiation and mineralization.

\section{Introduction}

Human bone tissue cells are mainly composed of osteoblasts, osteocytes and osteoclasts. Bone tissue maintains its homeostasis through a delicate dynamic balance between the bone-forming of osteoblasts (bone formation) and the bone-eroding of osteoclasts (bone resorption) ${ }^{[1]}$. This dynamic balance is often affected by mechanical load, nutrition, hormone levels and other factors ${ }^{[2,3]}$. In particular, mechanical loading plays an important role in bone tissue homeostasis. Frost's ${ }^{[4]}$ stress theory of bone stress, bone tissue physiological strain level 200-2500 $\mu \varepsilon$, but the bone can withstand $10000 \sim 30000 \mu \varepsilon$, of which $3000 \sim 5000 \mu \varepsilon$ is physiological overload strain, mechanical stress is greater than $5000 \mu \varepsilon$ stressor physiological long-term cyclic loading at overload strain levels, which is fatigue loading. In many cases, the bone tissue is in a mechanically overloaded environment, such as weight-bearing soldiers, highintensity training athletes and porter, etc ${ }^{[5-7]}$. They tend to bone pain, bone inflammation and other symptoms of fatigue bone damage. Therefore, the mechanical overload caused by fatigue of bone injury 
has become a threat to soldiers weight training and heavy physical activity of the normal medical problems.

It is well known that the Wnt signaling pathway plays a critical role in the regulation of bone formation. In recent years, many studies have confirmed that Wnt signaling pathway involved in the regulation of osteoblast biological response ${ }^{[8]}$. Considering the important role of Wnt signaling pathway in the process of osteoblast proliferation, differentiation and mineralization, we hypothesized that mechanical overload could regulate the proliferation, differentiation and mineralization of osteoblasts through influencing Wnt signaling pathway.

In this study, overload damage models were constructed by four-point bending mechanics. The effects of overload on osteoblasts and Wnt signaling pathway were detected by Western blot, immunofluorescence and laser confocal microscopy.

\section{Results}

\subsection{Mechanical overload on the proliferation of osteoblasts}

Mechanical strain is important for cell proliferative activity. The influence of mechanical overload strain on the proliferative activity of MC3T3-E1 cells was tested by MTT assay. MC3T3-E1 cells were applied loading with $5000 \mu \varepsilon$, which is above physiological range, applied once a day for 0.5 h over 3 consecutive days. As shown in Fig 1, compared with the blank control group, the mechanical overload group significantly inhibited cell proliferation.

\subsection{Mechanical overload on the differentiation of osteoblasts}

$\mathrm{COL} I$ and $\mathrm{OCN}$ are differentiation marker for the osteoblast. To see if mechanical overload strain modulates osteoblasts differentiation, MC3T3-E1 cells were subjected to mechanical overload strain. As shown in Fig 2A, the overloaded mechanical environment markedly down-regulated the mRNA expression of osteoblast differentiation marker gene, which was significantly different from the blank control group $(p<0.05)$, and bone matrix protein expression of COL I and OCN (Fig 2B) were also reduced by overload mechanical. Moreover, the effect was mediated partially via $\mathrm{Wnt} / \beta$-catenin signaling since the $\beta$-catenin and Runx 2 activation by overload mechanical was reduced (Fig 2).

\subsection{Mechanical overload on the impact of osteoblast skeleton}

Cytoskeleton plays a key role in maintaining cell movement and morphology, and is closely related to biological activities such as cell energy conversion, substance transport and mechanical signal transduction. In this study, cytoskeleton protein staining showed without any processing factors of osteoblast morphology typical triangular distribution; and the mechanical overload group cell shrinkage deformation, microfilament skeleton disorder, loose arrangement, poor direction and skeleton disruption (Fig 3). 


\subsection{Effect of mechanical overload on osteoblast mineralization}

The effect of mechanical overload on MC3T3-E1 cells mineralization was tested. MC3T3-E1 cells were exposed to overload with mechanical $5000 \mu \varepsilon, 0.5 \mathrm{~Hz}, 0.5 \mathrm{~h} /$ day for 3 days respectively. Osteoblasts were stained with Alizarinred $S$ at 14 days for the identification of mineralized nodules. As shown in Fig 4, There was a large amount of calcium nodules in the control group. Compared with the blank control group, the mechanical overload environment was obvious inhibited the formation of osteoblast calcium nodules.

\subsection{The effect of mechanical overload on $\beta$-catenin expression and nuclear translocation in Wnt signaling pathway}

After MC3T3-E1 cells were stretched, the protein expression of $\beta$-catenin was detected by immunofluorescence. It was found that mechanical overload environment significantly inhibited the expression of core factor $\beta$-catenin protein in Wnt signaling pathway and inhibited its transfer to nucleus (Fig 5), which was significantly different from the blank control group $(p<0.01)$.

\section{Discussion}

As we all know, bone tissue is the main organ of the human body to bear the mechanical load stimulation, it has the support, movement and protection function, and also plays an important role in the balance of mineral metabolism. A large number of studies have confirmed that the biological effects of bone cells (osteoblastic bone formation and osteoclastic bone resorption) through the reaction to adapt to different mechanical load environment ${ }^{[11,12]}$. Bone remodeling can maintain the stability of bone biomechanical properties, which is of great significance to improve bone strength ${ }^{[13]}$. Mechanical stimulation is one of the important factors that influence and control bone remodeling. Under physiological conditions, the bone is in the best mechanical environment. Bone formation and bone resorption are in a dynamic balance. At this time, the bone tissue is still in the stationary phase. When the mechanical microenvironment changes, the previous equilibrium state is broken, the osteoblastic bone formation and the osteoclastic bone resorption are adjusted accordingly, and finally a new balance is reached in the new mechanical environment. Osteoblasts are an important effector cell for bone remodeling, growth and repair, and are sensitive to changes in the surrounding mechanical load. It can feel the mechanical stimulation is converted into biological signals, thus regulating the bone tissue environment stability. In vivo, osteoblasts attached to the bone matrix in which the stress stimulation is extremely complex, subject to a variety of factors, so in vivo cell experiments are difficult to accurately reflect the mechanical load on the impact of osteoblasts. Therefore, in vitro experiments of osteoblasts in vitro loaded cells can effectively eliminate other interfering factors in the body, can accurately reflect the impact of overload mechanical stimulation on osteoblasts, which is helpful to further explore the effect of mechanical overload on bone tissue and its mechanism, and provide theoretical support for the prevention and treatment of bone tissue injury in extreme mechanical environment. 
In this study, osteoblasts were subjected to overload mechanical stimulation by four-point bending mechanical loading device to study the effect of overload mechanical environment on osteoblast proliferation, differentiation and mineralization, and to investigate the response mechanism of osteoblasts to overload mechanical signals. In this study, the proliferation of osteoblasts was examined by MTT colorimetric assay. The results showed that compared with the blank control group, mechanical overload stimulation significantly inhibited the proliferation of osteoblasts $(p<0.05)$. COL I is an important biomarker of early osteoblastic differentiation and bone formation, while $\mathrm{OCN}$ and calcium nodules are important biomarkers of late osteoblastic differentiation and maturation ${ }^{[14,15]}$. The results of real-time PCR showed that mechanical overload inhibited the expression of COL I and OCN osteoblast differentiation marker genes.Real-time PCR results were confirmed by Western Blot. The Western Blot results showed that overloading mechanical environment inhibited the expression of osteoblast differentiation marker protein. Alizarin red staining showed that it was difficult to find calcium nodule formation in the entire field of view of mechanical overload group. Cytoskeleton is a protein fiber network structure, which plays a key role in the maintenance of cell motility and morphology, and is closely related to biological activities such as cell energy conversion, substance transport and mechanical signal transduction ${ }^{[16]}$. In this study, cytoskeleton protein staining, the confocal microscope to observe the impact of mechanical overload on the cytoskeleton, the results showed that the osteoblasts of the blank group displayed typical triangular morphological distribution; and mechanical overload group cell wrinkle shrinkage deformation, disarrangement of microfilament arrangement, loose arrangement of skeleton, bad orientation and skeleton breakage.

The classical Wnt/ $\beta$-catenin signaling pathway plays a critical role in the development of bone and bone formation ${ }^{[17]}$. $\beta$-catenin protein is the core component of Wnt signaling pathway and plays an important role in osteoblast proliferation, differentiation and mineralization ${ }^{[18]}$. TCF/LEF, $\beta$-catenin and Smad4 polymerize to form complexes, which promote Smad4 into nucleus and mediate the transcription of Wnt target gene ${ }^{[19]}$. Therefore, the concentration of $\beta$-catenin in the cytoplasm determines Wnt signaling pathway closure and activation. When the Wnt signaling pathway was shut down, $\beta$-catenin was phosphorylated by GSK-3 $\beta$ to degrade $\beta$-catenin through the ubiquitin pathway, so that the concentration of $\beta$-catenin in the cytoplasm was maintained at a lower level. When the Wnt signaling pathway was activated, the Wnt protein specifically binds to the Frizzled receptor on the cell membrane surface, activating the protein DSH (Disheveled). The activated DSH can inhibited the degradation of $\beta$-catenin by GSK-3$\beta$, leading to $\beta$-catenin accumulates in the cytoplasm, thereby entering the nucleus and activating the TCF/LEF transcription factor, initiating transcription of the target gene ${ }^{[20]}$. In this study, Western Blot and immunofluorescence techniques were used to evaluate the protein content of $\beta$-catenin in the cytoplasm of osteoblasts. The results showed that the concentration of $\beta$-catenin in osteoblasts was maintained at low level after overload mechanical stimulation. Runx 2 is a kind of transcription factor protein, which can directly stimulates transcriptional expression of bone matrix protein genes such as COL I, OCN, and OPN in the process of osteoblast differentiation ${ }^{[21]}$. Thus, Runx 2 is also known as a promoter that integrates a variety of signals in turn affecting osteoblast differentiation ${ }^{\text {22] }}$. In this study, real-time PCR and Blot Western were used to evaluate the gene transcription level and protein expression 
of Runx 2. The results showed that the expression of Runx 2 was down-regulated by mechanical overload.

\section{Conclusions}

In summary, the experimental results showed overload mechanical stimulation reduced the expression of Runx 2 by acting on the classical Wnt/ $\beta$-catenin signaling pathway, thus inhibited osteoblasts proliferation, differentiation and mineralization. The study of the effect of overload mechanical stimulation on osteoblasts can further reveal the mechanism of overloaded bone injury and has important practical significance for the future prevention and treatment of fatigue bone injury in extreme overloaded mechanical environment.

\section{Materials And Methods}

\subsection{Cell culture}

MC3T3-E1 cells were purchased from the Institute of Basic Medical Sciences, Peking Union Medical College. The cells were maintained in alpha minimal essential medium (Gibco, USA) supplemented with $10 \%$ fetal bovine serum(Gibco, USA) and 1\% penicillin-streptomycin (Solarbio, China). The cells were cultured at $37^{\circ} \mathrm{C}$ and $5 \% \mathrm{CO}_{2}$. After reaching $90 \%$ confluence, the cells were detached by treatment with $10 \%$ trypsin-EDTA (Solarbio, China) and were seeded at the density of $2 \times 10^{4} / \mathrm{cm}^{2}$ in the cell culture dishes.

\subsection{Application of overload mechanical loading to cultured cells}

The stretching device was generated by a specially designed four-point bending device described previously ${ }^{[9]}$. The well-grown MC3T3-E1 cells were inoculated into the cell culture medium at a density of $2 \times 10^{4} / \mathrm{cm}^{2}$. After the cells were cultured for $24 \mathrm{~h}$, MC3T3-E1 cells were subjected to overload tensile mechanics stimulation with four-point bending mechanical loading device. The cell cultures were subjected to mechanical strain of $5000 \mu \varepsilon$ at $0.5 \mathrm{~Hz}$ for $0.5 \mathrm{~h}$ with this method of loading for three consecutive days.

\subsection{MTT assay cell proliferation}

The MTT assay was performed to determine the effects of Icariin on MC3T3-E1 cell viability. MC3T3-E1 cells will be continually cultured for $24 \mathrm{~h}$ after mechanical stimulation, and then the proliferation was detected. After $24 \mathrm{~h}$ culture, cells were washed with phosphate-buffered saline (PBS, Solarbio, China), then $1 \mathrm{ml}$ of MTT solution (Sigma, USA; $5 \mathrm{mg} / \mathrm{ml}$, diluted by PBS) was added to each well and incubated for $4 \mathrm{~h}$ at $37^{\circ} \mathrm{C}$. After $4 \mathrm{~h}$, the supernatant was removed, then $2 \mathrm{ml}$ of Dimethyl sulfoxide (Sigma, USA) was added to all wells and mixed thoroughly to dissolve the dark blue crystals. After 10 min at room temperature to ensure that all crystals were totally dissolved, the absorbance was measured at $490 \mathrm{~nm}$ by a microplate reader (Tecan, Switzerland). 


\subsection{The cytoskeleton of the laser confocal microscopy}

MC3T3-E1 cells will be continually cultured for $24 \mathrm{~h}$ after mechanical stimulation, and then the changes of cytoskeleton were observed under confocal microscope. After incubation for $24 \mathrm{~h}$, the cells were washed with $4 \%$ paraformaldehyde (Solarbio, China) for $10 \mathrm{~min}$ and then with $0.5 \%$ Triton- 100 solution for $5 \mathrm{~min}$. The TRITC-labeled phalloidin working solution (Sigma, USA) was incubated in the dark at room temperature for $30 \mathrm{~min}$. Finally, a certain amount of DAPI solution (Solarbio, China) was added for $1 \mathrm{~min}$. After treatment, the cells were observed under a laser scanning confocal microscope (Nikon, Japan).

\subsection{RNA isolation and real-time RT-PCR}

The real-time PCR was performed with the real-time quantitative analyzer (Bio-rad IQ5, USA). The mRNA expression of osteoblast differentiation related gene COL I, OCN, Runx 2, and $\beta$-catenin were detected by real-time PCR. The primer sequences are shown in Table 1. The operating conditions were as follows: predenaturation at $95^{\circ} \mathrm{C}$ for $3 \mathrm{~min}$, denaturation at $95^{\circ} \mathrm{C}$ for $15 \mathrm{~s}$, annealing at $60^{\circ} \mathrm{C}$ for $1 \mathrm{~min}$, cycling at 40 cycles and cooling at $4^{\circ} \mathrm{C}$. Each sample was repeated 3 times for assay analysis.

Table 1. Osteoblastic differentiation marker gene primer sequences

\begin{tabular}{|lll|}
\hline Gene (mouse) & Forward & Reverse \\
\hline OCN & CCATCTTTCTGCTCACTCT & ACTACCTTATTGCCCTCCT \\
\hline COL $\square$ & CAGTGGCGGTTATGACTT & CTGCGGATGTTCTCAATCT \\
\hline Runx 2 & GCAGCACTCCATATCTCTAC & GCGTCAACACCATCATTCT \\
\hline -catenin & GCCATCTGTGCTCTTCGTCA & TGGTGGGTGCAGGAGTTA \\
\hline GAPDH & CAGCAACTCCCACTCTTC & TGTAGCCGTATTCATTGTC \\
\hline
\end{tabular}

\subsection{Western blot}

Each culture was rinsed with PBS twice and solubilized with radio immunoprecipitation assay buffer (Beyotime, China ). Protein concentration was determined with the Micro BCA Protein Assay Reagent Kit (Solarbio, China). Approximately $35 \mu \mathrm{g}$ proteins were subjected to sodium dodecyl sulfate polyacrylamide gelelectrophoresis (Solarbio, China) to determine CoL I, OCN, Runx 2 and $\beta$-catenin. Subsequently, the proteins were transferred onto PVDF membrane (Solarbio, China). The blots were blocked by incubation in $5 \%$ milk with TBST (Beyotime, China) for $1 \mathrm{~h}$ and probed overnight at $4{ }^{\circ} \mathrm{C}$ with rabbit anti-CoL I, rabbit anti-Runx 2, rabbit anti-OCN and rabbit anti- $\beta$-catenin (Beyotime, China), respectively. After washing, the membranes were incubation with HRP-conjugated appropriate secondary antibody (Beyotime, China囚 1:5000 in 5\% milk) for 60 min at room temperature. The immunoreactive bands were visualized using enhanced chemiluminescence detection kit (Beyotime, China). Optical density of the protein bands was determined with Gel Doc 2000 (Bio-Rad, CA, USA). The expression of GAPDH (Beyotime, China) was used 
as a loading control and data were normalized against those of corresponding GAPDH. Results were expressed as relative to control.

\subsection{Mineralization Assay}

The cultures were stained as described by Majors et al ${ }^{[10]}$. MC3T3-E1 cells will be continually cultured for $14 \mathrm{~d}$ after mechanical stimulation, and then the proliferation was detected. After $24 \mathrm{~h}$ cultured, cells were washed with PBS and fixed with $4 \%$ paraformaldehyde for $15 \mathrm{~min}$ at room temperature. Then use the deionized water or PBS to wash the plate three times. After washing, alizarin red S (Solarbio, China) was used to incubate room temperature with mild shaking for $30 \mathrm{~min}$ (the time may be adjusted by the cells) to stain the calcium nodes. The plate was washed with PBS three times and each time 5 min, then cells were visualized under light microscope (Japan, Nikon).

\subsection{Statistical analysis}

All experiments were performed in triplicate and repeated at least three times. Data were presented as mean \pm standard deviation (Mean $\pm S D$ ). Group differences were assessed by one-way analysis of variance (ANOVA) followed by Scheffe's test. Statistical analysis was performed using SPSS 19.0 software. Avalue of $p<0.05$ was considered to be significant.

\section{Declarations}

\subsection{Consent for publication}

All authors agreed to publish

\subsection{Availability of data and materials}

The data and materials in the paper are available

\subsection{Competing interests}

The authors have no potential conflicts of interest in this article.

\subsection{Acknowledgements}

This work was supported by the Tianjin Science and Technology Project (19ZXDBSY00070), the National Natural Science Foundation of China (11972198), and the Special Project of Tianjin Clinical Medicine Key Discipline (HWZX001).

\subsection{Authors' contributions}

Yaru Fan, Yingbin Yan conceived and designed the experiments. Han Liu, Rui Luo performed the cell experiment. Ruixin Li carried out the data analysis. Yingjie Liu drafted the manuscript. Hao Liu revised the 
manuscript. All authors read and approved the final manuscript.

\section{Contributor information}

Yaru Fan, E-mail: 17862196077@163.com.

Yingbin Yan, E-mail: yingbinyan@qq.com

Han Liu, E-mail: liuhan75628@163.com

Rui Luo, E-mail: Iuoruiwf@163.com

Ruixin Li, E-mail: limxinxin@163.com.

Yingjie Liu, E-mail: 659296168@qq.com.

Hao Liu, E-mail: Kqlh2013@163.com.

\section{References}

[1] Al Bari A A, Al Mamun A. Current advances in regulation of bone homeostasis[J]. FASEB BioAdvances, 2020,2(11):668-679.

[2] Wang Q, Duan M, Liao J, et al. Are Osteoclasts Mechanosensitive Cells?[J]. J Biomed Nanotechnol, 2021,17(10):1917-1938.

[3] Krishnan A, Muthusami S. Hormonal alterations in PCOS and its influence on bone metabolism[J]. J Endocrinol, 2017,232(2):R99-R113.

[4] Frost H M. Bone "mass" and the "mechanostat": a proposal[J]. Anat Rec, 1987,219(1):1-9.

[5] Qu H, Yu L J, Wu J T, et al. Spine system changes in soldiers after load carriage training in a plateau environment: a prediction model research[J]. Mil Med Res, 2020,7(1):63.

[6] Pihlajamaki $\mathrm{H}$, Parviainen $\mathrm{M}$, Kyrolainen $\mathrm{H}$, et al. Regular physical exercise before entering military service may protect young adult men from fatigue fractures[J]. BMC Musculoskelet Disord, 2019,20(1):126.

[7] Rothenberg P, Grau L, Kaplan L, et al. Knee Injuries in American Football: An Epidemiological Review[J]. Am J Orthop (Belle Mead NJ), 2016,45(6):368-373.

[8] Burgers T A, Williams B O. Regulation of Wnt/beta-catenin signaling within and from osteocytes[J]. Bone, 2013,54(2):244-249.

[9] Tang $L$ L, Wang $Y L$, Pan J, et al. The effect of step-wise increased stretching on rat calvarial osteoblast collagen production[J]. J Biomech, 2004,37(1):157-161. 
[10] Majors A K, Boehm C A, Nitto H, et al. Characterization of human bone marrow stromal cells with respect to osteoblastic differentiation[J]. J Orthop Res, 1997,15(4):546-557.

[11] Islander $U$, Jochems $C$, Stubelius $A$, et al. Combined treatment with dexamethasone and raloxifene totally abrogates osteoporosis and joint destruction in experimental postmenopausal arthritis[J]. Arthritis Res Ther, 2011,13(3):R96.

[12] Lewiecki E M. Combination therapy: the Holy Grail for the treatment of postmenopausal osteoporosis?[J]. Curr Med Res Opin, 2011,27(7):1493-1497.

[13] Pogoda P, Priemel M, Rueger $\mathrm{J} \mathrm{M}$, et al. Bone remodeling: new aspects of a key process that controls skeletal maintenance and repair[J]. Osteoporos Int, 2005,16 Suppl 2:S18-S24.

[14] Lee H S, Jung EY, Bae S H, et al. Stimulation of osteoblastic differentiation and mineralization in MC3T3-E1 cells by yeast hydrolysate[J]. Phytother Res, 2011,25(5):716-723.

[15] Duarte W R, Shibata T, Takenaga K, et al. S100A4: a novel negative regulator of mineralization and osteoblast differentiation[J]. J Bone Miner Res, 2003,18(3):493-501.

[16] Hohmann T, Dehghani F. The Cytoskeleton-A Complex Interacting Meshwork[J]. Cells, 2019,8(4).

[17] Houschyar K S, Tapking C, Borrelli M R, et al. Wnt Pathway in Bone Repair and Regeneration - What Do We Know So Far[J]. Front Cell Dev Biol, 2018,6:170.

[18] Nusse R, Clevers H. Wnt/beta-Catenin Signaling, Disease, and Emerging Therapeutic Modalities[J]. Cell, 2017,169(6):985-999.

[19] Mulholland D J, Dedhar S, Coetzee G A, et al. Interaction of nuclear receptors with the Wnt/betacatenin/Tcf signaling axis: Wnt you like to know?[J]. Endocr Rev, 2005,26(7):898-915.

[20] Cao Z, Fu Y, Sun X, et al. Aluminum trichloride inhibits osteoblastic differentiation through inactivation of Wnt/beta-catenin signaling pathway in rat osteoblasts[J]. Environ Toxicol Pharmacol, 2016,42:198-204.

[21] Wang Y, Sun J, Zhang Y, et al. Stichopus japonicus Polysaccharide Stimulates Osteoblast Differentiation through Activation of the Bone Morphogenetic Protein Pathway in MC3T3-E1 Cells[J]. J Agric Food Chem, 2021,69(8):2576-2584.

[22] Ziros P G, Basdra E K, Papavassiliou A G. Runx2: of bone and stretch[J]. Int J Biochem Cell Biol, 2008,40(9):1659-1663.

\section{Figures}




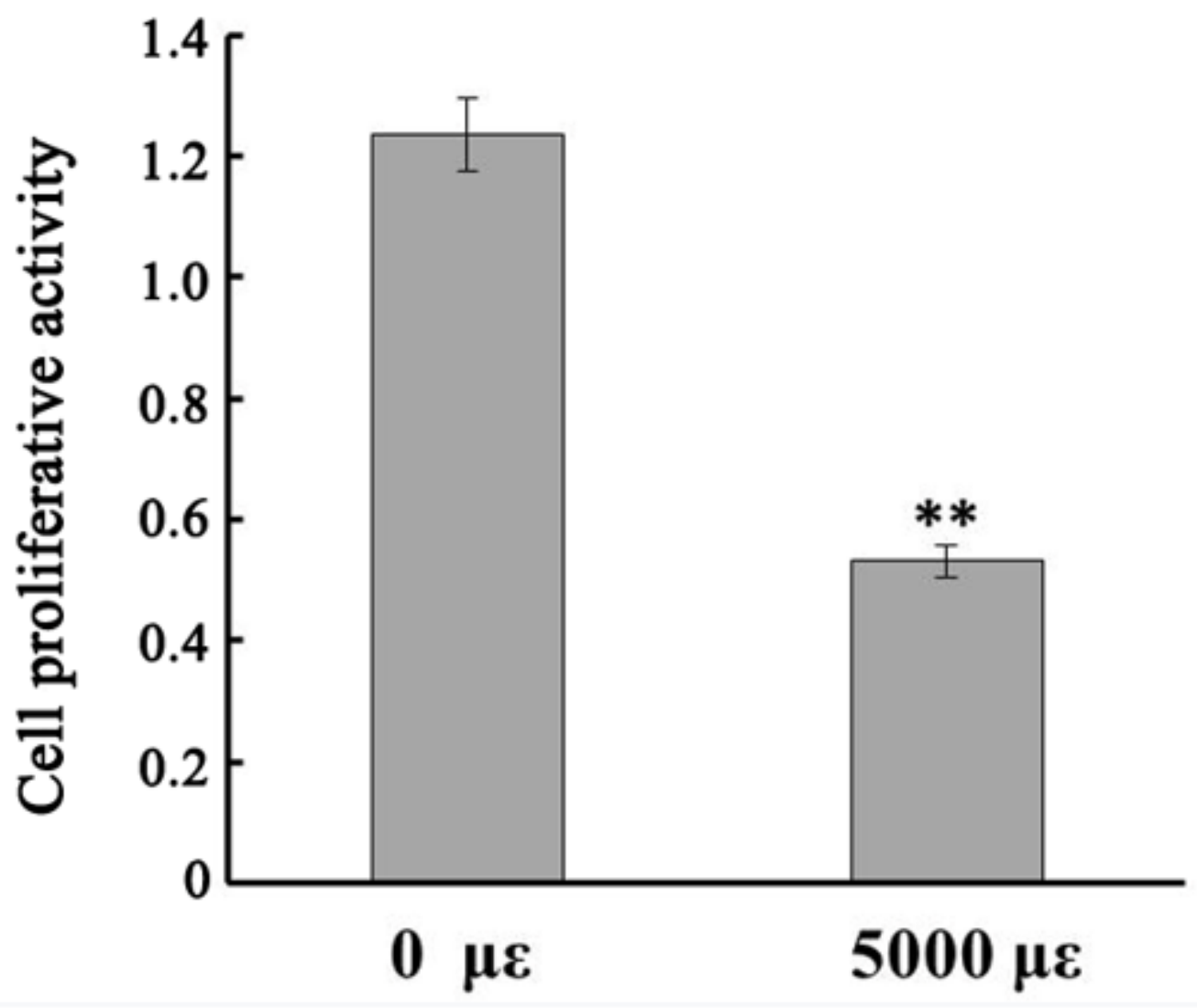

Figure 1

Effect of mechanical overload on osteoblastsproliferation. The proliferation was evaluated by MTT assay under mechanical overload strains of $5000 \mu \varepsilon$ at $0.5 \mathrm{~Hz}$, once a day for $0.5 \mathrm{~h}$ over 3 consecutive days. The results are expressed as Mean \pm SD of three independent experiments. ${ }^{\star} p<0.05,{ }^{\star \star} p<0.01$, Vs. the control group. 


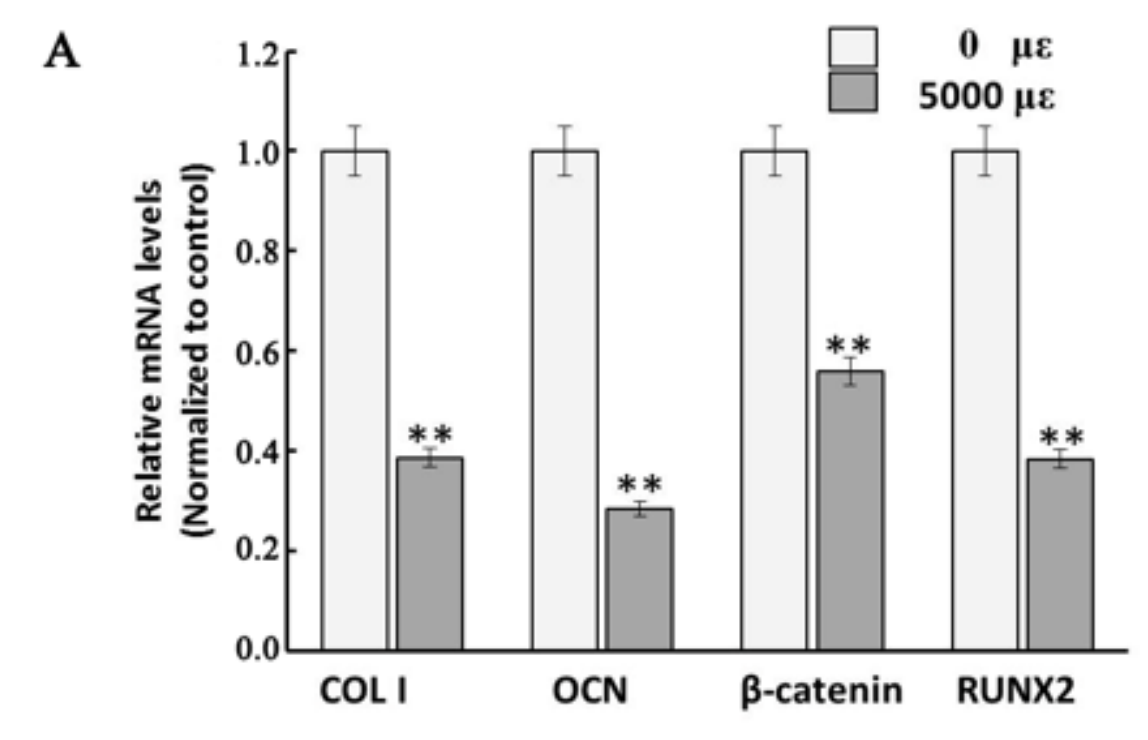

B

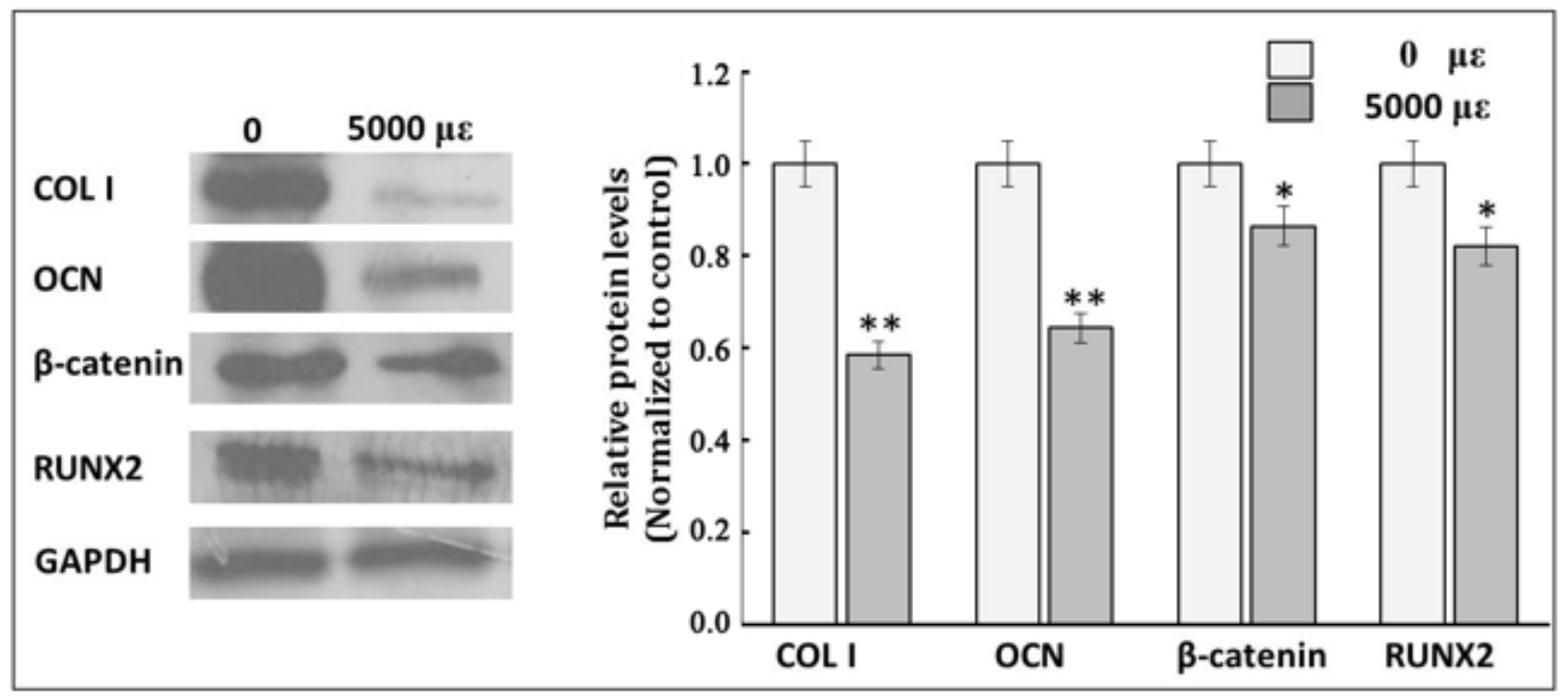

Figure 2

Effect of mechanical overload on the expression of osteoblasts differentiation related genes and proteins. A) The mRNA expressions of CoL I, OCN, $\beta$-catenin and Runx 2 in MC3T3-E1 cells were determined by real-time PCR under mechanical overload strains of $5000 \mu \varepsilon$ at $0.5 \mathrm{~Hz}$, once a day for $0.5 \mathrm{~h}$ over 3 consecutive days. B) The protein expressions of $\mathrm{COL}$ I, OCN, $\beta$-catenin and Runx 2 in MC3T3-E1 cells were determined by Western-blot under mechanical overload strains of $5000 \mu \varepsilon$ at $0.5 \mathrm{~Hz}$, once a day for $0.5 \mathrm{~h}$ over 3 consecutive days. GAPDH was used as an internal control. All data represent the Mean \pm SD of at least three biological replicates, ${ }^{\star} p<0.05,{ }^{*} p<0.01$, Vs. the control group. 


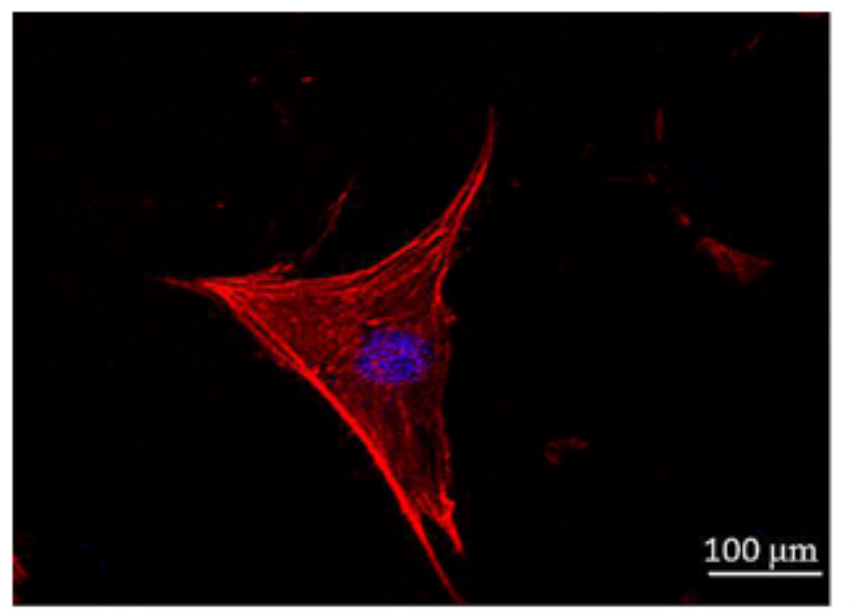

$0 \mu \varepsilon$

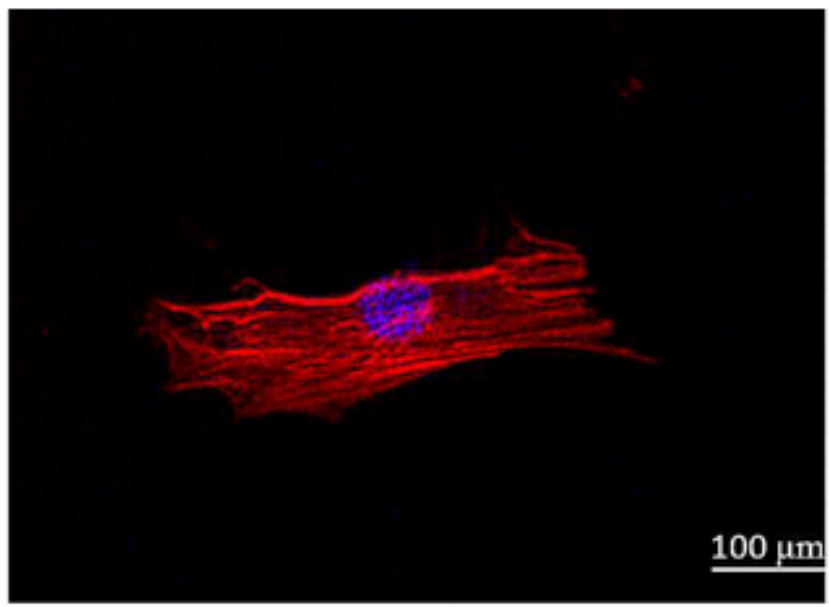

$5000 \mu \varepsilon$

\section{Figure 3}

Effects of mechanical overload on the cytoskeleton of osteoblasts. The MC3T3-E1 were exposed mechanical overload strains of $5000 \mu \varepsilon$ at $0.5 \mathrm{~Hz}$, once a day for $0.5 \mathrm{~h}$ over 3 consecutive days, followed by the immunofluorescence for actin filament (red) using specific fluorescent dyes. The nuclei were stained with DAPI (blue), and the changes of cytoskeleton were observed under laser scanning confocal microscope, scale bar, $100 \mu \mathrm{m}$. The figure is representative data from three to five independent experiments.

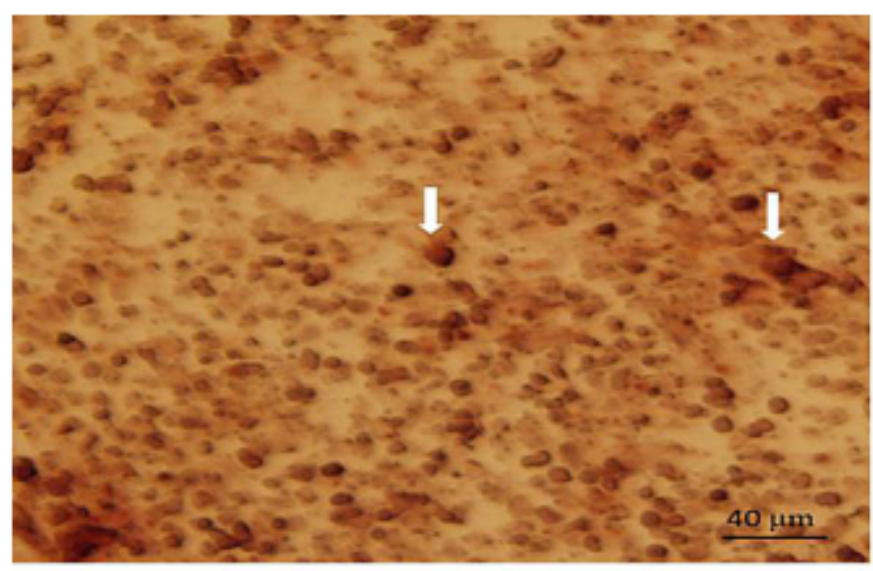

$0 \mu \varepsilon$

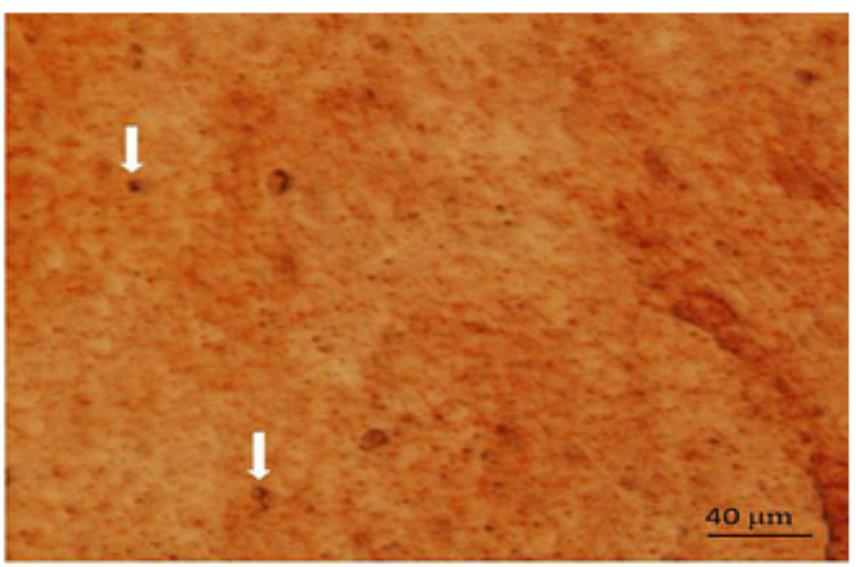

$\mathbf{5 0 0 0} \boldsymbol{\mu \varepsilon}$

\section{Figure 4}

Effects of mechanical overload on the mineralization of osteoblasts. Mineralization of MC3T3-E1 cells was determined by Alizarinred S staining. MC3T3-E1 cells were firstly cultured in osteogenic differentiation medium for 7 days, and then under mechanical overload strains of $5000 \mu \varepsilon$ at $0.5 \mathrm{~Hz}$, once a day for $0.5 \mathrm{~h}$ over 3 consecutive days. MC3T3-E1cells were stained with Alizarinred S at 14 days for the 
identification of mineralized nodules, Scale bar, $40 \mu \mathrm{m}$. The figure is representative data from three to five independent experiments.
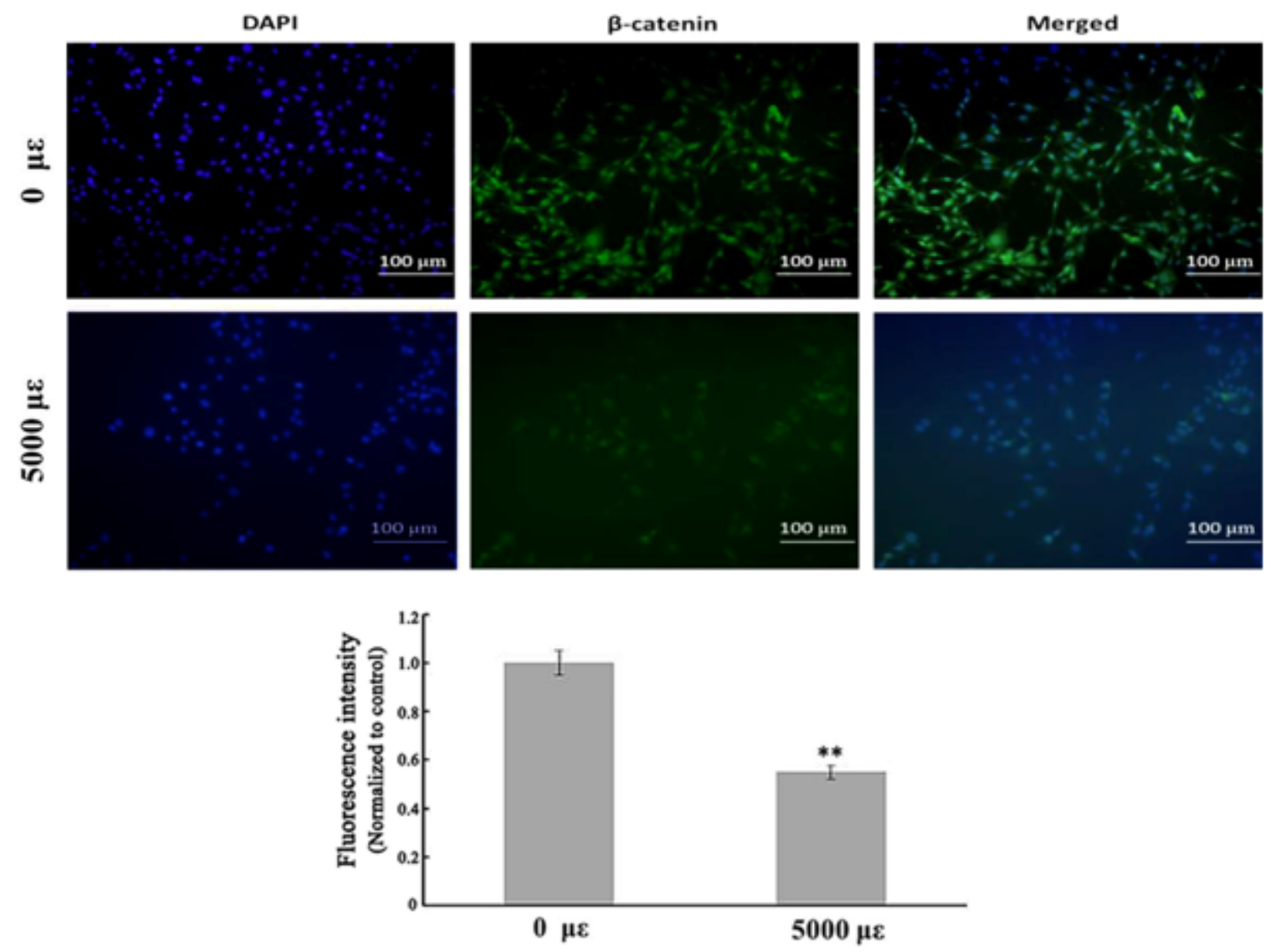

Figure 5

Effect of mechanical overload on the Wnt/ $\beta$-catenin signalingpathway inMC3T3-E1 cells. The MC3T3-E1 were exposed mechanical overload strains of $5000 \mu \varepsilon$ at $0.5 \mathrm{~Hz}$, once a day for $0.5 \mathrm{~h}$ over 3 consecutive days, followed by the immunofluorescence for $\beta$-catenin (green) using anti- $\beta$-catenin. The nuclei were stained with DAPI (blue), scale bar, $100 \mu \mathrm{m}$. The results are expressed as the mean \pm SD of three independent experiments. ${ }^{*} p<0.05,{ }^{*} p<0.01$, Vs. the control group. 